\title{
Inventare una pelle per tutto: traduzione italiana di un estratto de Cujo, di Nuno Ramos
}

Irma Caputo *

\section{Introdução}

O seguinte texto é a tradução de um trecho do livro de Nuno Ramos Cujo (1993, p. 19-37) para o italiano, que nasce como acompanhamento de uma pesquisa de doutorado na área de tradução do livro $O$ (2008) do mesmo autor. A pesquisa, além de uma análise sobre a visão de linguagem emergente da obra de Nuno Ramos e sua interseção com as práticas das artes-plásticas, parte, para a tradução, de uma análise dos elementos formais recorrentes da prosa poética do autor. Portanto na tradução do seguinte texto foram levados em conta: aspectos da prosa poética como reiteração de células sonoras, tais que rimas, assonâncias, consonâncias e aliterações, especialmente as com afinidades semânticas. Tentou-se reproduzir no estilo de escrita uma das técnicas que autor parece mutuar das artes plásticas, a justaposição, que, em entrevista inédita (abril 2017), ele define com "limites borrados".

\section{Tradução}

Inventare una pelle per tutto.

Ho passato l'asfalto freddo sulla pece, scurendola.

Somigliava a una lama oleosa altamente tossica. Poi, con un pennello ho cosparso la pece sciolta sull'asfalto freddo per farlo seccare. Il risultato è stato una specie di gomma minerale e smagliante che ricopriva in modo strano il feltro che le stava sotto. Ora avevo un pezzo di qualcosa. Dovevo metter su qualcosa, darvi una forma, ma non sapevo come determinarla questa forma. Non so perché, qualsiasi scelta mi sembrava falsa. Volevo venisse da sé. Quindi ho solo messo insieme vari pezzi e li ho cuciti in un tappeto informe. I contorni di questo tappeto, tuttavia, sembravano sempre scelti con cura. Alla fine ho distrutto tutto. Non so andare al di là della pelle.

Stamattina pioveva come se fosse così da sempre. Ho guardato oltre la finestra e ho visto piccioni dalle ali pesanti e cani tristi. Ho preso l'impermeabile e sono uscito. Il caffellatte mi riscaldava. Questa pioggia, ho pensato, uguale a quella di ieri e a quella di domani, è tutto ciò che ti succederà oggi. Approfittane, allora.

Ho bisogno di dimenticare la felicità, ma non al punto di essere infelice. Soltanto al punto di dimenticare la felicità.

\footnotetext{
* Mestrado em Literatura e Cultura Comparada (Português-Alemão) pela Universidade Orientale de Nápoles com ênfase em tradução literária e estudos pós-coloniais. Atualmente doutoranda da Puc Rio da linha de pesquisa "Linguagem, sentido e tradução" orientanda do poeta e tradutor Paulo Henriques Britto com um projeto de tradução do livro Ó (2008) do artista plástico Nuno Ramos.
} 
Oggi ho visto una lucertola. Non una lucertola, una foglia che sembrava una lucertola. Non una foglia, una pietra che sembrava una foglia. Dunque è una pietra, ho pensato distrattamente.

Immaginare ogni dettaglio. Un gancio, per esempio, se si deve appendere qualcosa. Il tetto, per esempio, se il gancio è attaccato al tetto. Una casa, se si tratta del tetto di una casa, o il cielo se si sta a cielo aperto.

L'informe, infine, si organizza per i bordi.

In mezzo all'amorfo, forme rette.

Mi fermo a fissare il sole in un raggio di sole, il piccione in un volo di piccione, il bianco del mattone in un mattone bianco. Mi ammalerei se non immaginassi le cose come in uno scenario: un impasto d'argento liquido in cui si riflettono parole e con esse dettagli decontestualizzati (memorie, piccoli anagrammi incomprensibili agli altri), come fossero cose oltre che immagini, con proprietà fisiche che le facciano affondare (piccole reminiscenze, o simboli, o storie personali, o barzellette prive d'umore) nell'argento, nel piombo, che ribolle come uno specchio, lasciando una cicatrice física del loro passaggio nel mondo (finalmente, sono affondate).

Volti conosciuti, poco a poco, si concentrano in un unico volto.

C'è uno stagno fatto di parole cosparse d'olio, stampate col gesso, ma non riesco a leggerle; una musica piena d'altre musiche; animali morti e la sensazione che non si tratti di uno scenario. Lì, qualcosa mi aspetta. Un senso profondo che per questo deve interrompere tutti gli altri sensi superficiali, imponendosi e trasformando (ecco la paura) il passato in sciocchezze (in versioni insufficienti rispetto alla nuova). Qualcosa sta per essere detto (quando, da chi?): una versione trionfale che mostrerà il fracasso completo delle altre. Non posso smettere di crederci. E se non dovessi crederci più, peggio ancora: quest'ultima sentenza, già severa, si trasformerà in un unico momento del grande fracasso. Sarà anch'essa insufficiente e passeggera (e per questo ancora ottimista), contaminata negativamente dal grande regolamento di conti. Il terrore si apre così, come un carciofo, a strati successivi, verso un centro rimandato ad ogni avanzo. Ogni rivelazione non è altro che l'infanzia del terrore futuro e totale.

Non devo completare tutto. Stare in pari con se stesso è una forma di avarizia. Ho bisogno di trovare la frazione corretta di fracasso.

Meglio trasformare l'angustia in un teatro e l'orrore in uno scenario, piuttosto che diventare "una persona sofferente".

Si dice di quelli che non sono mai usciti: non sono mai usciti, come se questo fosse un fatto. Di quelli che sono usciti non si dice nulla. $\mathrm{O}$ si dice che sono usciti, come se fossero stati aiutati a diventare ciò che sono diventati.

Io volevo vederlo, ma non lo vidi. Io volevo averlo, ma non lo ebbi. Io volli. Io volevo dio, ma non l'ho avuto. Io volevo l'uomo, il figlio, il primo animale, ma non ho potuto vederli. Stavo steso, sveglio. Stavo così sin dall'inizio. Volevo muovermi, ma 
non mi mossi. Io volli. Stavo di spalle, morto sin dall'inizio. L'erba alta non mi faceva vedere. Morto sin dall'inizio. Io volevo la paura, ma non ho potuto averla. Stavo steso, di spalle proprio morto. Volevo vedere il primo animale e la radice della prima pianta. L'erba alta non mi faceva vedere. Volevo restare sveglio, ma dormii.

Stavo steso e l'erba alta non mi faceva vedere. Gli occhi fuori dalle orbite quasi morivano per l'ultima volta. Stavo lì sin dall'inizio. Io volevo la paura, ma non ho potuto averla. Volevo il sonno, la cassaforte, qualche algoritmo romano. Volevo l'uomo, ma non questo qui. Ascoltai mille rumori senza sapere cosa fossero. Stavo di spalle sull'erba. Volevo girare il corpo e guardare il cielo, ma non questo qui. Volevo guardare la carne, da dove comincia, sotto la pelle, ma non troppo in profondità. Volevo guardare la carne e la radice della prima pianta (questa aveva solo lo stelo). Volevo la paura, ma non quella lì. Volevo dire: quella lì. Volevo girare il corpo senza muovermi. Morto sin dalla prima pianta. Morto, proprio morto, da dove comincia la prima pianta. Ero un fossile della prima pianta, ma non di quella pianta lì. Volevo dire: quella pianta lì. Volevo guardare, guardare e guardare questo qui. Stavo di spalle sull'erba alta senza muovermi. Volevo girare il corpo per vedere il cielo, ma non questo qui. Ero proprio morto e ho voluto dire proprio questo qui.

La pelle del coniglio senza il coniglio dentro: i peli penetrano il cuoio passando per attraverso piccoli pori rosati. Ci sono colori diversi per ogni pelo, ma non molti: semitoni tra il giallo e il castano scuro e anche alcuni albini. È così senza il coniglio dentro.

Interrato in terra, elevato in cielo.

Ho cominciato a strappare la pelle dalle cose. Volevo vedere cosa vi fosse sotto. Ho alzato la superficie del pavimento, è venuta via intera, senza rompersi. Ho dovuto sbucciare la pelle ai mattoni, poco a poco, con pazienza. La pelle del cemento era la più sottile di tutte e quella delle maioliche rifletteva fatto specchio. Sotto a queste pelli sembrava esserci un'altra pelle, identica, e tuttavia, rugosa. Ne tolsi un altro strato e la superficie rugosa era aumentata. Tolsi poco a poco gli strati successivi, sempre più ondulati e accidentati. La pelle delle doghe del pavimento, per prima, aveva mostrato grandi rombi e una tonalità rossastra emergeva nella parte inferiore. Piccole schegge di legno ora vi si attaccavano sopra, perforandola in più punti. Gli strati di pelle del cemento cominciarono ad attaccarsi gli uni agli altri. Ormai non era più possibile toglierli così sottili (quasi trasparenti) e la forza impiegata cominciò ad aumentare (avevo le braccia stanche, adesso). L'alterazione più triste è stata quella della pelle delle maioliche: quanto più profondi, tanto più opachi diventavano gli strati. La nitidezza speculare della prima pelle (molto più forte di quella dell'intera maiolica) si era trasformata poco a poco in una tonalità lattiginosa di un giorno nuvoloso o di un occhio cavato. La pelle dei mattoni man mano si trasformava in polvere: se all'inizio era ancora possibile staccarla, adesso aveva perso ogni consistenza disintegrandosi al primo tocco. Non era più pelle, né superficie: si era trasformata in un materiale arenoso qualsiasi. Poteva essere polvere di mattone, calce, sabbia o, chissà, i resti di un defunto. Dietro ad ogni pelle, dunque, incontravo appena forme degradate della pelle superficiale. Nonostante i dati non siano sufficienti, devo concludere che questo primo strato non ricopre un interno diversificato, ma è l'espressione più stabile di questo interno, che la ripete monotonamente. 
Gli specchi sono acqua. Tutto ciò che riflette è ancora umido.

Due forme di instabilità: la brillantezza (moltiplicazione di una superficie) e l'umidità (degradazione o evoluzione della superficie originale in un'altra). La scultura prenderà corpo in questi due modi, ossia: attraverso la sua possibilità di degradazione $o$ di evoluzione e della sua capacità di riflettere, di ricevere l'apparenza di un'altra superficie. Inoltre, al massimo, la scultura deve durare appena un istante. Se dura per sempre, non dura per nessuno.

\section{L'afflizione davanti alle cose che durano. Per chi durano?}

Su un numero, il numero. Su una pietra, un granello di sabbia: la pietra, il granello di sabbia. Su una sfera (tra tante): la sfera, tra tante. Su un suono, nella sinfonia: questo suono, nella partitura. Su una stella: l'unica stella, sui greci questo greco, sui rospi questo rospo. Sulle piogge: questa pioggia, questa goccia. Sui simboli: nessun simbolo, sulla memoria: nessuna, nessuna.

Morti, vivi. Cani, latrati.

Asciugare i fiammiferi.

Cucina e beve il suo rancore tutte le notti. Si fa una ferita alla pelle e si lamenta con gemiti costanti mentre tira le crosticine perché il taglio non cicatrizzi (è difficile tagliarsi alla dimensione giusta, la ferita deve rimanere stazionaria: non può estendersi fino a farla ammalare davvero, ma nemmeno rimarginarsi al punto da eliminare il pretesto dei suoi gemiti. Il risentimento, il rancore e l'invidia attecchiscono in un grande senso di proporzione e misura). Dice addio a ciò che non è mai successo e in un paesaggio deserto, arido, asessuato, dove persino i cammelli muoiono, in un'Arabia senza dio, lei si domanda: come ho potuto fare ciò? Como ho potuto gettar via la mia unica possibilità? e la risposta evidente per tanto spreco è: è stato lui, o: è stata colpa loro, o: io non avevo soldi, loro ce li avevano, o, ancora più difficile: loro non sanno quanto valgo, non sanno cosa si perdono. Così, sollevata, riprende fiato per un'altra stagione in questo inferno. L'odio che non si esterna, la rabbia insopportabile di non aver vissuto, si ritorce senza tregua contro il suo portatore (gli si invecchia dentro, invecchiandolo). Per liberarsene distilla il suo rancore, con calma, in un pomeriggio di pioggia. Riesce in buona parte a risparmiarsi, ma deve sacrificare troppe cose: in verità, deve sacrificare l'intero mondo, infatti non identificando un bersaglio preciso contro cui scagliarsi, trasforma tutta l'eterogeneità, le mezze tonalità in un unico grande bersaglio, un unico colpevole unificato. Ma, come un fantasma, di notte, lui ritorna a tirarle i piedi.

RAMOS, Nuno. Cujo. Rio de Janeiro: editora 34, 1993, p. 19-37.

Data de envio: 25-09-2017

Data de aprovação: $12-12-2017$

Data de publicação: 22-12-2017 